\title{
Histopathologic Evaluation of three Ultraviolet-Activated Composite Resins on Monkey pulps
}

\author{
R. J. Heys, D. R. Heys, C. F. Cox and J. K. Avery \\ Departments of Operative Dentistry and Oral Biology, Dental Research Institute, \\ University of Michigan, Ann Arbor, Michigan 48109, U.S.A.
}

\begin{abstract}
The pulpal responses of three ultraviolet-activated composite resins, Nuva-Fil ${ }^{\circledR}$, Experimental UV \#1 ${ }^{\circledR}$ and Experimental UV \#2 ${ }^{\circledR}$, were tested on adult monkey teeth using silicate and zinc oxide eugenol (ZOE) as positive and negative controls. All materials were placed in Class $\mathrm{V}$ cavity preparations in Rhesus monkey teeth using approximately 48 anterior and 63 posterior teeth of both the maxillary and mandibular arches. A total of 111 teeth were utilized and all materials were evaluated at 3 days, 5 and 8 weeks. Following left ventricular perfusion, the teeth were prepared for microscopic evaluation using routine histological procedures. The 3-day pulpal response of all the ultraviolet-activated composites was slight with some disruption and vacuolization in the odontoblastic layer and a slight inflammatory response. At 5 weeks there was a reduction of the inflammatory response and the formation of reparative dentin was noted for all ultraviolet composites. The 8 -week pulp response was slight, characterized by a minimal inflammatory response adjacent to the zone of reparative dentin. Generally, ZOE produced the mildest response while silicate produced the most severe response at the three time intervals.
\end{abstract}

Received 10 January, accepted for publication 9 March 1977

The introduction and use of dental restorative materials that are polymerized by exposure to ultraviolet activation was first reported by Buonocore (1970). He used these materials as sealants in association with an enamel etch technique. Since that time their use has broadened to include repair of cervical erosion and abrasion, repair of incisal angles, the direct bonding of orthodontic brackets, restoration of Class III cavities and in some instances Class I cavities. As a restorative material, the obvious advantage of such a system is in the operator's ability to control the working time. This allows for ease in placement and contouring, with as much time as is needed. Upon completion of this procedure the operator can activate the ultraviolet light source and polymerize the material.

Stanley et al. (1972) reported on the pulpal response of an ultraviolet light-cured material in monkey teeth, comparing it with a self-curing resin and zinc oxide eugenol material (IRM - Intermediate Restorative Material). They found that at 3 days postoperatively, all materials exhibited a similar degree of minimal pulp response, however, focal hemorrhage occurred in $80 \%$ of the teeth restored with the ultraviolet-cured resin. As the post-operative time increased to 60 days the ultraviolet-cured resins exhibited persistent or increasing pulpal responses as compared with either the selfcuring composite resin or with zinc oxide 
eugenol. They concluded that the use of this material necessitates placement of a calcium hydroxide liner to minimize the pulpal responses observed at 60 days post-operative. They speculated that this persistent and intensifying pulp response may be due to incomplete polymerization of the resin resulting in the continued penetration of these irritating agents.

It is the purpose of this study to evaluate in detail the pulpal compatibility of two newly-formulated ultraviolet-activated resins and further to compare them to a commercially available ultraviolet-activated resin according to the guidelines suggested by the American National Standards Committee MD-156 for Dental Materials and Devices (1972).

\section{Materials and Methods}

After a thorough prophylaxis, cavities were prepared in 111 adult Rhesus monkey teeth which consisted of approximately 48 anterior and 63 posterior teeth. Class V cavities were prepared by high speed air turbine (200,000-300,000 RPM) with water and air spray using \#35 inverted cone burs. The cavity depths were achieved by penetration of the bur to one-and-a-half times the height of the bur head. This resulted in approximately $0.5-0.8 \mathrm{~mm}$ remaining dentin. The width of the cavity was twice that of the bur head.

Placement of the materials was predetermined by random selection, making certain that each compound was placed in both anterior and posterior teeth of both arches. Each of the three resin compounds was placed in nine teeth for each test period while the controls were placed in five teeth. All materials were evaluated at three time periods: 3 days, 5 weeks and 8 weeks as recommended by the American National Standards Committee. MD-156 (1972).

The compounds used in this study were
Nuva-Fil ${ }^{\circledR 1}$, Kerr's Experimental UV\# ${ }^{\circledR 2}$, and Experimental UV \#2 ${ }^{\circledR 3}$, Ultravioletactivated resins with $\mathrm{ZOE}\left(\mathrm{Cavitec}^{\circledR}\right)^{4}$ and Silicate $\left(\mathrm{MQ}^{\circledR}\right)^{5}$ as controls as recommended by the ADA standards to ensure comparative evaluation. Two ultraviolet light sources were used to cure the ultraviolet-activated resins. The Nuvalite ${ }^{\circledR 6}$ was used to cure the Nuva-Fil ${ }^{\circledR}$ material and the Kerr Photo Light ${ }^{\circledR 7}$ was utilized to activate the two experimental ultraviolet resins for $1 \mathrm{~min}$ each.

After 3 days, 5 or 8 weeks the monkeys were sacrificed. All animals were initially sedated with an intramuscular injection of $0.8 \mathrm{cc}$ of Ketamine ${ }^{\circledR}$ into the quadriceps muscles. Deep anesthesia was acquired by intravenous administration of approximately $1.0-1.5 \mathrm{cc}$ of sodium pentobarbitol $(25 \mathrm{mg} /$ cc) providing a profound anesthesia and muscle relaxant. The monkeys were then perfused through the left ventricle and their bodies flushed with $0.9 \%$ physiologic saline, followed by a 20 min flush with an alcohol formalin acetic acid (AFA) fixative. The teeth were surgically removed from the jaws, decalcified, embedded in paraffin, serially sectioned and stained with hematoxylin and eosin.

In an attempt to be objective, tissue sec-

1 Nuva-Fil ${ }^{\circledR}$ Ultraviolet Activated Resin, L. D. Caulk Co., Milford, Delaware.

2 Experimental UV \#1 ${ }^{\circledR}$ Ultraviolet Activated Resin (\#201-87-1) Kerr Mfg. Co., Romulus, Michigan.

3 Experimental UV \#2 ${ }^{\circledR}$ Ultraviolet Activated Resin (\#201-93-1), Kerr Mfg. Co., Romulus, Michigan.

4 Cavitec $^{\circledR}$ (Batch \#01541020), a Zinc Oxide Eugenol Liner, Kerr Mfg. Co., Romulus, Michigan.

5 Silicate $\mathrm{MQ}^{\circledR}$ (Batch \#36607 liquid, \#1666602 - powder) Silicate Cement. S. S. White, Philadelphia, Penn.

6 Nuvalite $^{\circledR}$ - L. D. Caulk Co., Milford, Delaware.

7 Kerr Photo Light ${ }^{\circledR}$ - Kerr Manufacturing Company, Romulus, Michigan. 
tions were evaluated by the investigators prior to their knowledge of the specific materials used. All histological findings were evaluated according to Stanley's et al.'s (1967) and Mjör \& Tronstad's (1972) criteria, that is the general classification of slight, moderate and severe.

A Slight Reaction is characterized by a slightly increased cellularity in the cell-free zone adjacent to the cavity tubules. Some of these cells are typical inflammatory cells, mainly granulocytes. A mild congestion is found in the pulp tissue subjacent to the cavity tubules. Small irregularities in the odontoblastic layer are found, often associated with a displacement of odontoblastic nuclei into the dentinal tubules. The predentin may be reduced in width.

A Moderate Reaction is characterized by a slightly increased cellularity of granulo- cytes; histiocytes are seen adjacent to the cut tubules. A localized congestion with occasional hemorrhages is seen in the odontoblastic and adjacent pulpal region. The odontoblastic layer is discontinuous, with many odontoblastic nuclei displaced into the dentinal tubules. The predentin is generally reduced in width or may be absent.

A Severe Reaction is characterized by marked cellular infiltrates, mainly granulocytes, in the pulp tissue subjacent to the cavity tubules. Localized abscess formation may also be observed. Signs of congestion may be found surrounding the cellular infiltration, and hemorrhages may be seen. The odontoblastic layer is disrupted or destroyed, with many odontoblastic nuclei displaced into the dentinal tubules. The predentin is severely reduced in width, and is often absent altogether.

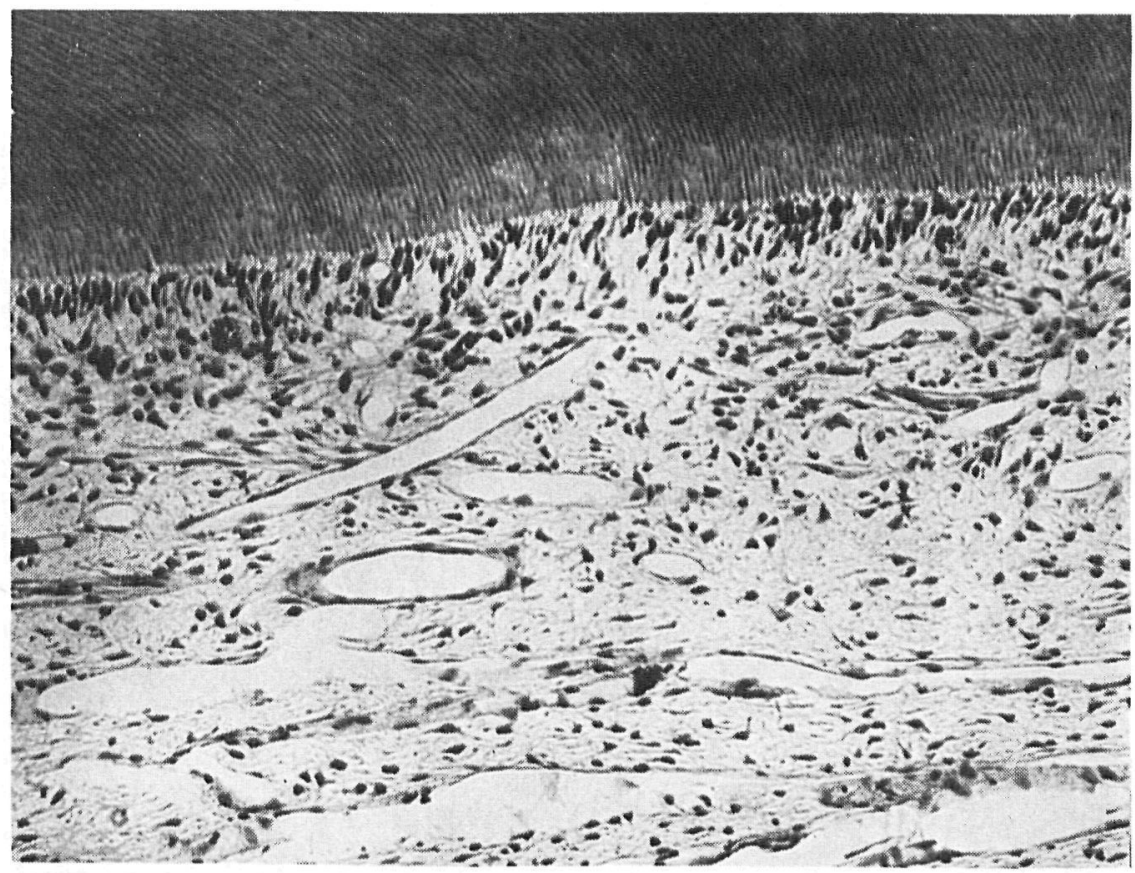

Fig. 1. ZOE - 3 day response. This is a slight response characterized by some disruption of the odontoblast layer with loss of its columnar appearance beneath the cavity preparation. Inflammatory response is slight. $\times 160$. 

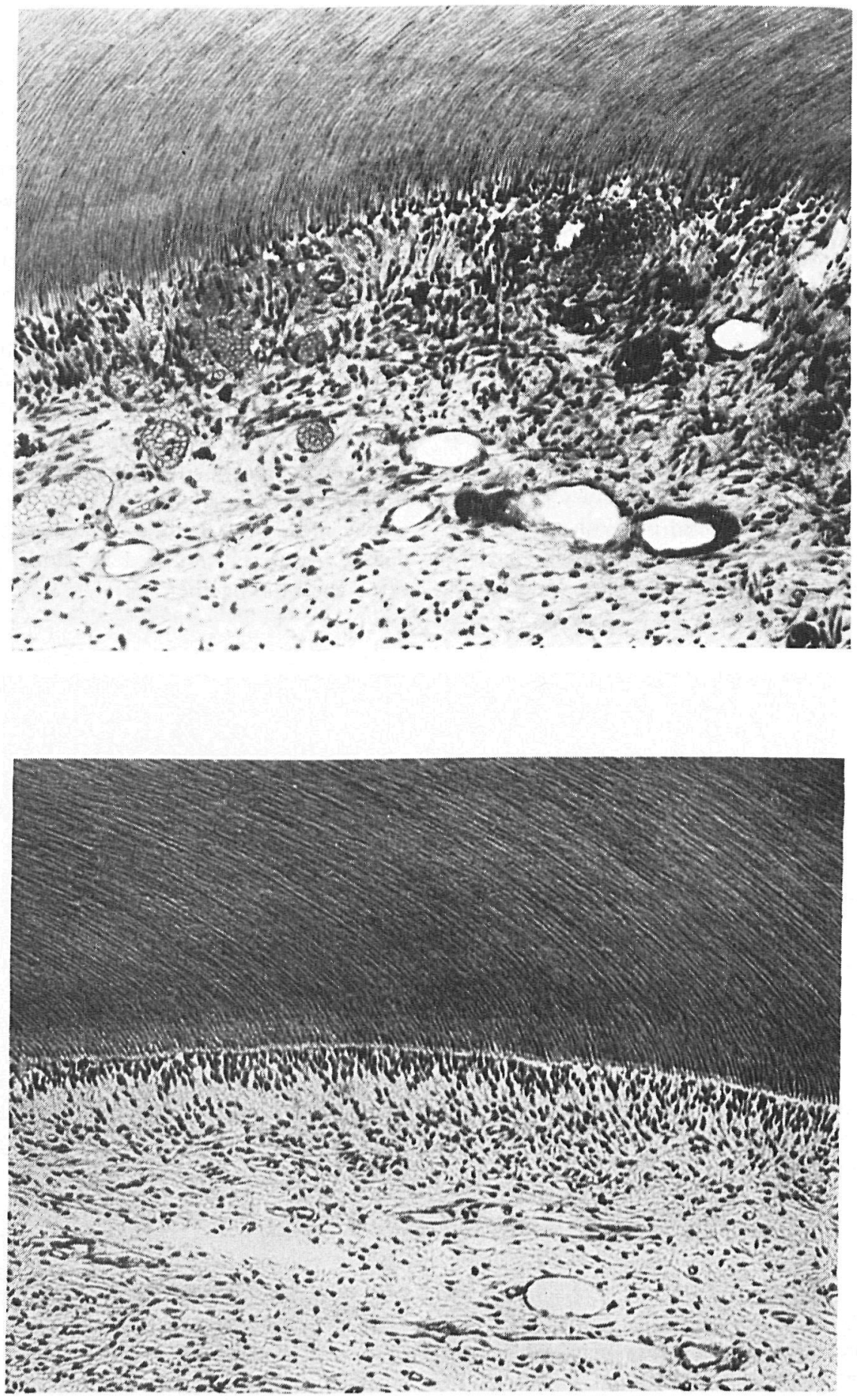


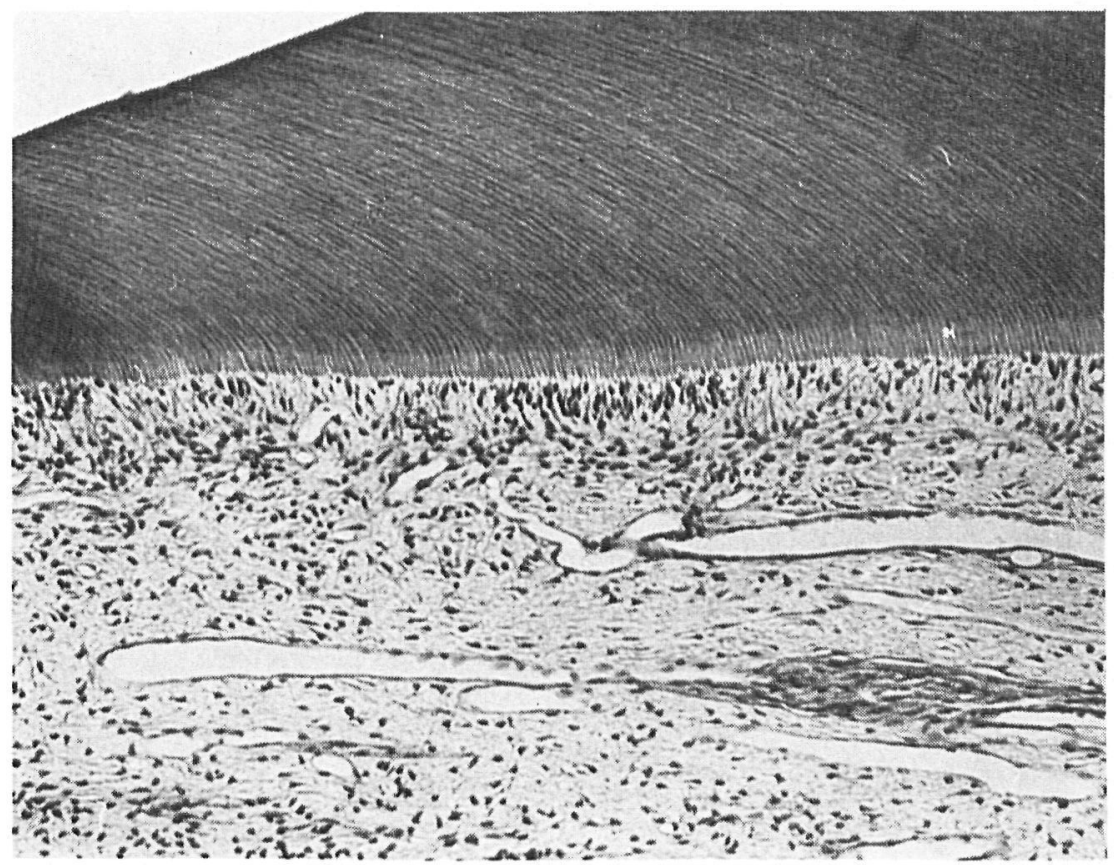

Fig. 4. Experimental UV \#2 ${ }^{\circledR}-3$ day response. The response underlying this deep cavity preparation shows some disruption and the loss of the columnar appearance of the odontoblasts. There is a slight increase in cellularity due to the presence of inflammatory cells. $\times 123$.

\section{Results}

\section{Day}

At the 3-day time interval ZOE (Fig. 1) (Cavitec ${ }^{\circledR}$ ) produced a slight response characterized by minimal cellular disruption, slight vacuolization of the subodontoblastic layer, and a very slight inflammatory response. The Silicate (Fig. 2) exhibited a more severe response characterized by a marked increase in the disruption of the odontoblastic region, some areas of vacuolization and displacement of odontoblastic nuclei into dentinal tubules with a moderate infiltration of inflammatory cells to the subjacent tissue. Nuva-Fil ${ }^{\circledR}$, Experimental UV \#1 ${ }^{\circledR}$ and the Experimental UV \#2 ${ }^{\circledR}$ (Figs. 3 and 4) all produced a slight response at 3 days. The response was characterized by some disruption of the odontoblastic layer with evidence of vacuolization and the presence of aspirated nuclei. The cell-free zone was lost due to increased cellularity; however, there was a minimal inflammatory response present. The effected areas were immediately under the cavity preparation

Fig. 2. Silicate -3 day response. This is a moderate response characterized by disruption of the odontoblastic layer, displacement of nuclei into the tubules, loss of the cell-free zone, congestion of the blood vessels and a moderate inflammatory response. $\times 102$.

Fig. 3. Nuva-Fil -3 day response. The response is slight and is restricted to the area underlying the cavity preparation. Minimal disorganization of the odontoblastic zone and the inflammatory response is graded slight. $\times 135$. 

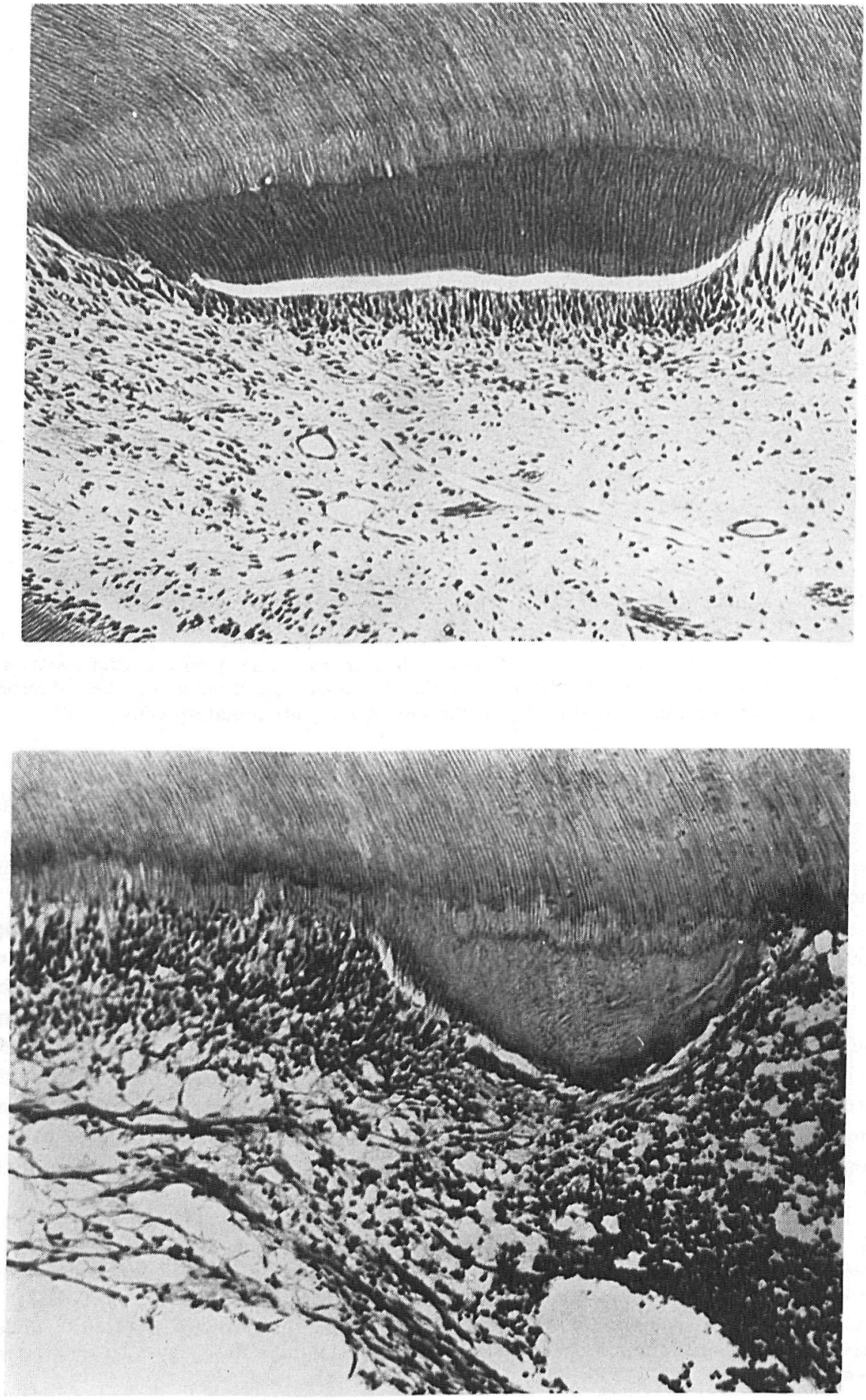


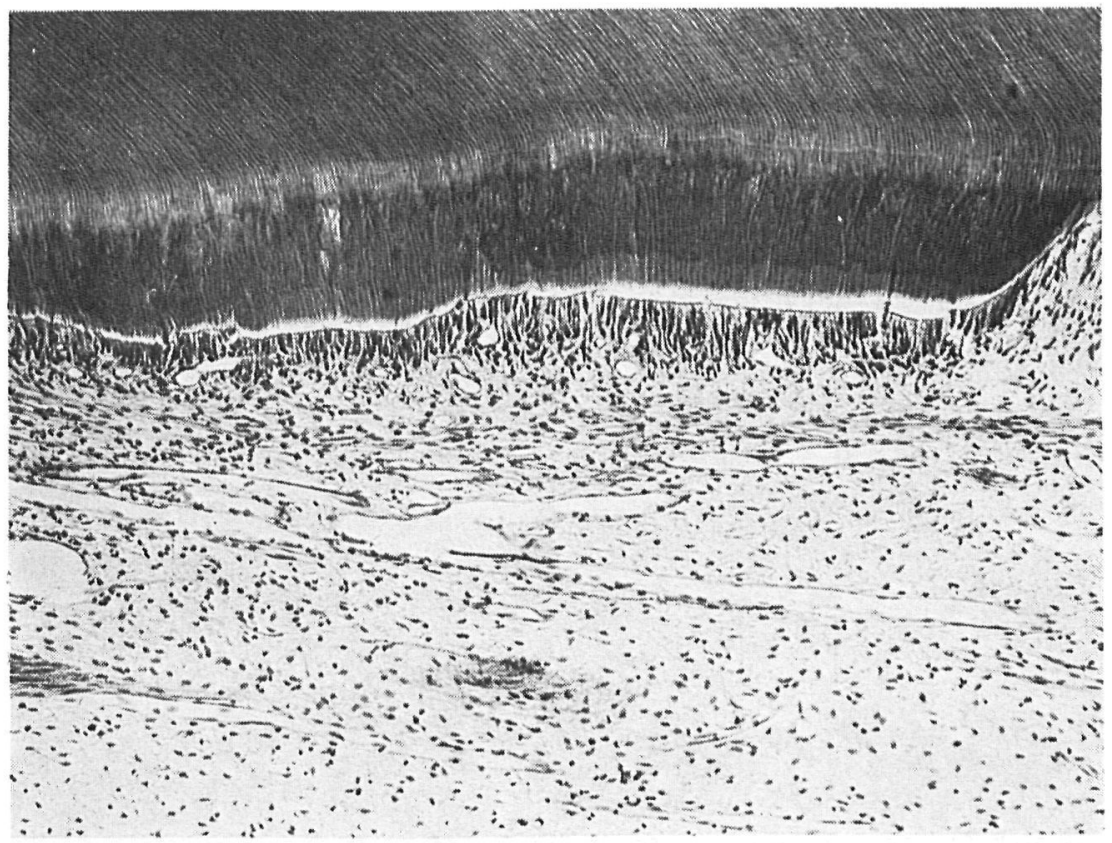

Fig. 7. Nuva-Fil ${ }^{\circledR}-5$ week response. A slight response is seen as characterized by reparative dentin beneath the cavity preparation. The odontoblastic layer is intact, a small amount of inflammatory cells is still present near odontoblastic layer. $\times 90$.

while the remaining pulp tissue appeared normal.

\section{Week}

As the post operative time increased to 5 weeks, ZOE (Cavitec ${ }^{\circledR}$ ) elicited only a slight response (Fig. 5). The reparative dentin underlying the cavity preparation was characterized by the reestablishment of a predentin border and the reorganization of a columnar odontoblastic layer. The presence of inflammatory cells was minimal. Silicate at 5 weeks presented varied responses from moderate to severe. In the moderate response, reparative dentin was present under the cavity preparation with the reorganization of an intact odontoblastic layer. In the more severe responses (Fig. 6), irregular reparative dentin was seen and the adjacent odontoblastic layer showed complete disorganization and vacuolization with much disruption of the subjacent pulp tissue. This was usually characterized by focal necrosis and abscess formation affecting a large proportion of the coronal pulp. At 5 weeks, Nuva-Fil ${ }^{\circledR}$, Experimental UV \# $1^{\circledR}$ and Ex-

Fig. 5. ZOE - 5 day response. Uniform reparative dentin is present beneath the cavity preparation and a uniform zone of predentin can be seen. The odontoblastic layer is intact, and few if any inflammatory cells can be found. $\times 135$.

Fig. 6. Silicate -5 week response. A severe response with reparative dentin formed beneath the cavity preparation, disruption and loss of the odontoblastic layer and areas of focal necrosis and abscess formation. $\times 125$. 

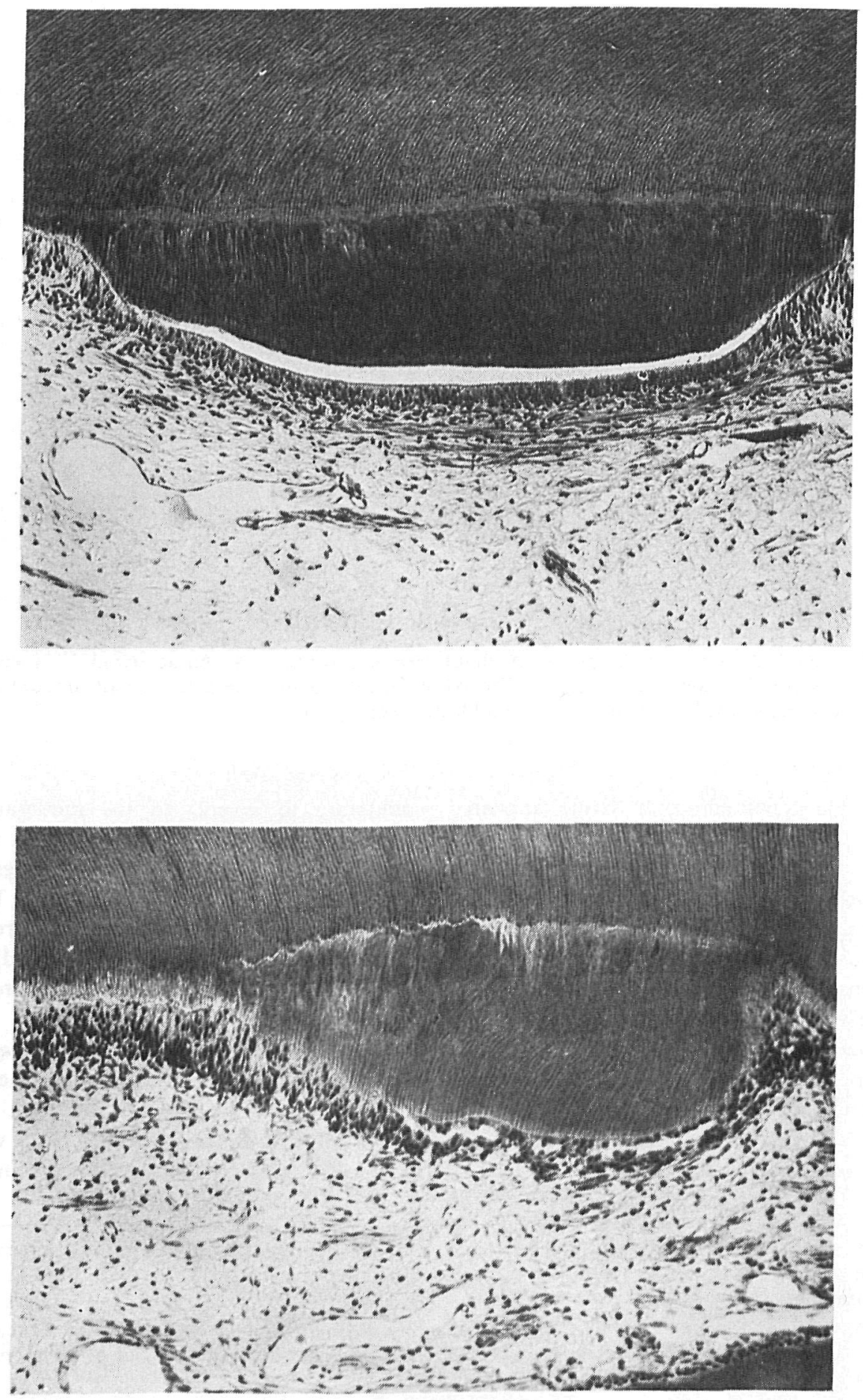


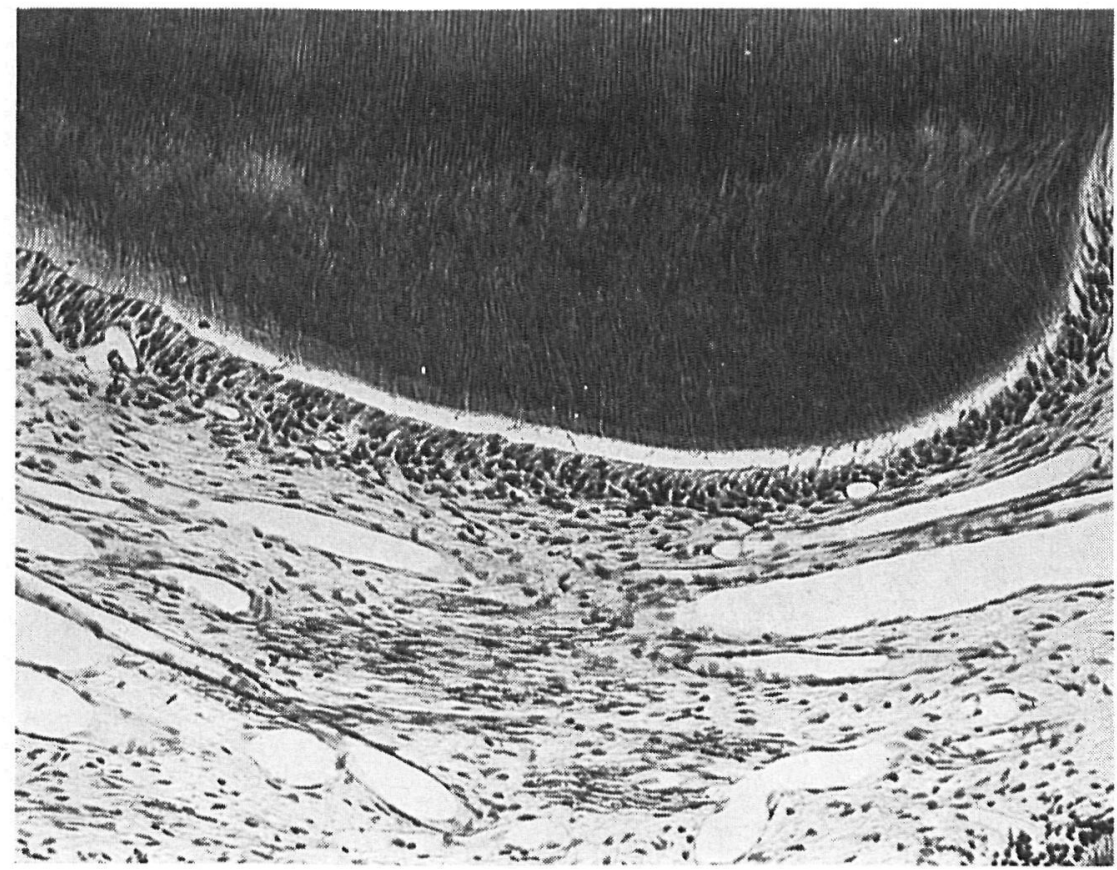

Fig. 10. Nuva-Fil ${ }^{\circledR}-8$ week response. A large area of reparative dentin is located under the cavity preparation, a uniform odontoblastic layer is present, and a slight inflammatory response is noted. $\times 140$.

perimental UV \#2 ${ }^{\circledR}$ materials all produced a slight response similar to that seen at 3 days (Fig. 7). Reparative dentin present beneath the cavity preparation was generally tubular in nature with some areas of irregularity and entrapped cells seen in the initial portion of the reparative dentin. The odontoblastic layer had reestablished itself underlying a thin layer of predentin but the cell-free zone was absent and a few inflammatory cells were still present.

Reparative and remaining dentin measure- ments at 5 weeks indicated a varying amount of reparative dentin and remaining dentin. Fig. 12 indicates that cavity preparations in posterior teeth were more shallow (a greater amount of remaining dentin) than those in anterior teeth; however, the amount of reparative dentin formed was generally similar for both anterior and posterior teeth. It should also be noted that Nuva-Fil ${ }^{\circledR}$ stimulated slightly more reparative dentin formation than did either Experimental UV \# $1^{\circledR}$ or the Experimental UV\# $2^{\circledR}$.

Fig. 8. ZOE - 8 week response. This slight response is characterized by a zone of reparative dentin beneath the cavity preparation, the odontoblastic layer is present and intact and a few chronic inflammatory cells still persist. $\times 115$.

Fig. 9. Silicate -8 week response. A thick zone of reparative dentin is present underlying the cavity preparation; the odontoblastic layer is reestablished along with a predentin border. The inflammatory response is minimal in this case. $\times 120$. 


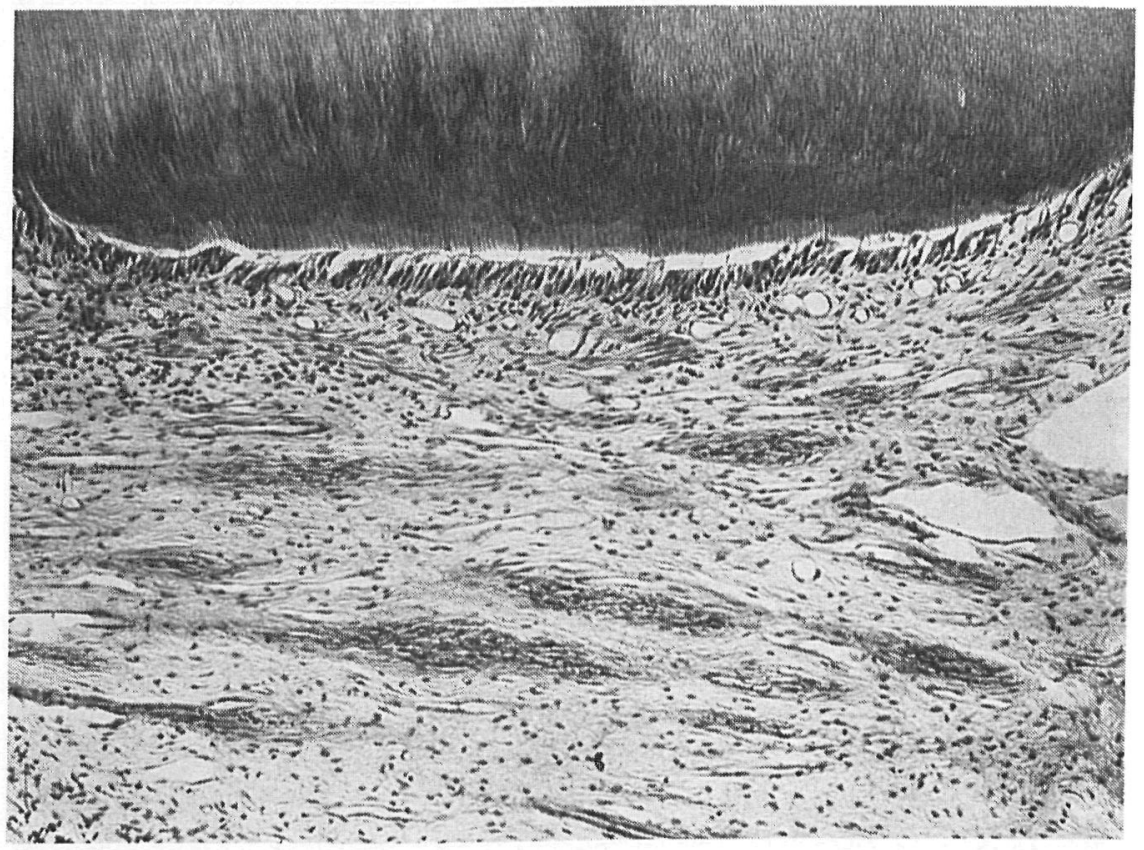

Fig. 11. Experimental UV \#1 ${ }^{\circledR}-8$ week response. A slight response characterized by a zone of reparative dentin beneath the cavity preparation. A slight cellular increase is noted in the area due to a few inflammatory cells present at this time. The response is graded slight. $\times 105$.

\section{Week}

The 8-week response of zinc oxide eugenol was slight to none (Fig. 8). The amount of reparative dentin formed was slightly greater than noted at 5 weeks with the reestablishment of the predentin zone and an intact odontoblastic layer. The remaining pulpal tissue appeared normal with little inflammatory response. Silicate at this time showed a greater variation, as well as severity of response than any of the other materials tested (Fig. 9). Some specimens exhibited a moderately severe reaction characterized by continued disruption and loss of the odontoblastic layer, the presence of chronic inflammatory cells and in some cases focal areas of necrosis and abscess formation. In the less severe response the odontoblastic layer overlying the reparative dentin was intact with only occasional areas of dis- ruption. Silicate produced almost twice as much reparative dentin as that seen at 5 weeks, with a continued persistence of chronic inflammatory cells.

As noted in the 3-day and 5-week time periods, the pulpal response of Nuva-Fil ${ }^{\circledR}$, Experimental UV \# ${ }^{\circledR}$ and Experimental UV\# $2^{\circledR}$ resins were again similar and characterized as a slight response (Figs. 10 and 11). The 8-week response was typified by an increase in the amount of reparative dentin as compared to the 5-week response. The odontoblastic layer appeared intact and continuous but the juxtaposed cell-free zone was still absent. There were few if any inflammatory cells present and the remaining pulp tissue appeared normal.

Measurements of both reparative and remaining dentin were also taken at the 8-week time interval (Fig. 13). Reparative 
GRAPH ।

COMPARISON OF REMAINING

TO REPARATIVE DENTIN

FIVE WEEK INTERVAL

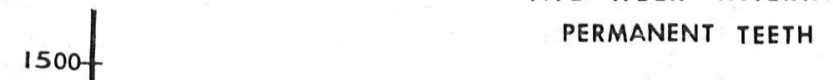

REMAINING DENTIN

REPARATIVE DENTIN
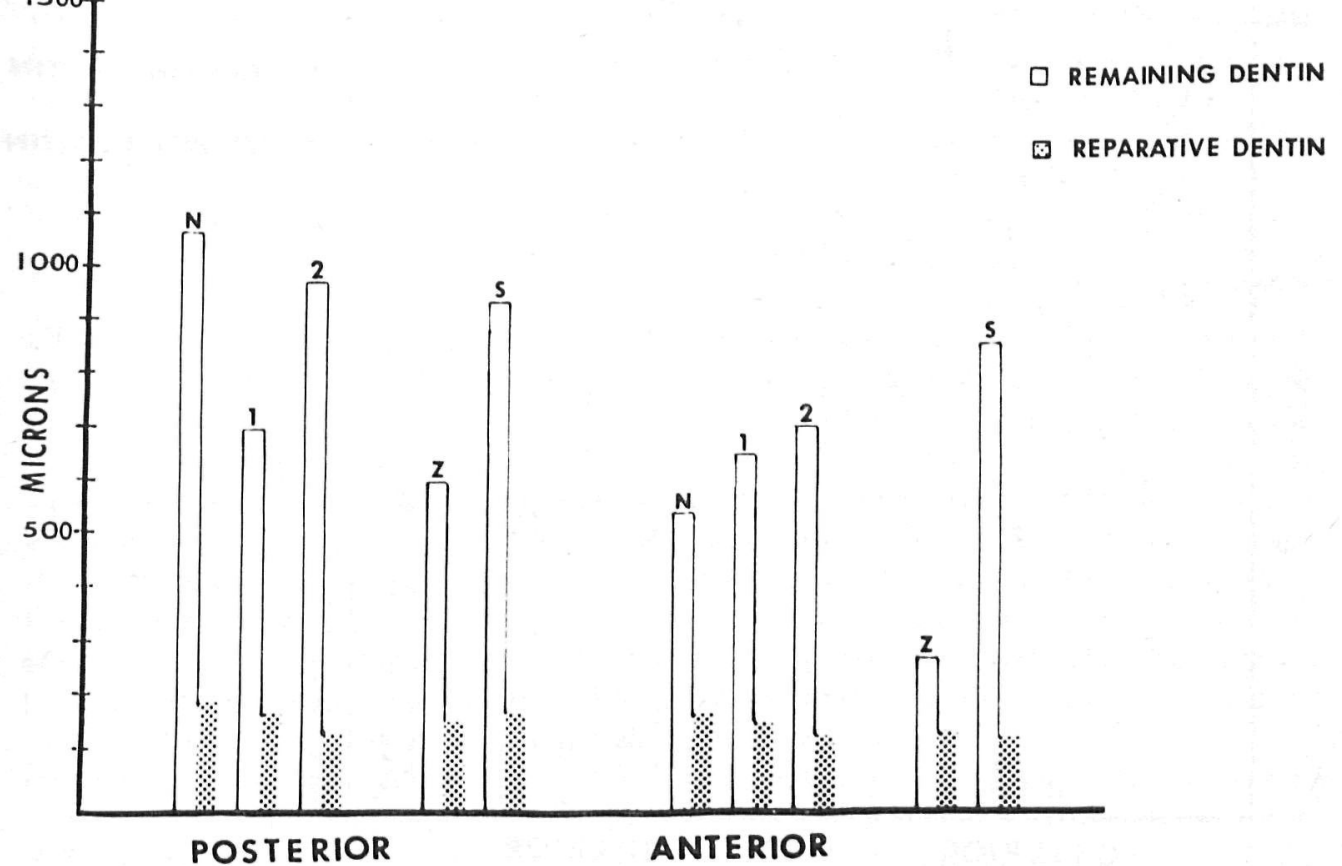

$N=$ Nuva-Fil ${ }^{\circledR}$

$1=$ Experimental U. V. \#1 ${ }^{\circledR}$

$2=$ Experimental U. V. \#2 ${ }^{\circledR}$

$Z=Z O E\left(\right.$ Cavitec) ${ }^{\circledR}$
$S=$ Silicate

dentin measurements indicated a slight in-

UV \#1 ${ }^{\circledR}$ and Experimental UV \# $2^{\circledR}$, a crease in the amount of dentin from the fact also observed at 5 weeks.

5 - to the 8 -week interval. Our measurements tended to show the cavity preparations in posterior teeth leave more remaining dentin (more shallow cavity depth) than that noted in anterior teeth. But as was noted at 5 weeks, the amount of reparative dentin formed for both anterior and posterior teeth appeared similar regardless of the amount of remaining dentin, with silicate being the exception at this time period. At 8 weeks Nuva-Fil ${ }^{\circledR}$ continued to produce more reparative dentin than Experimental

\section{Discussion}

It was our intention in this study to evaluate Nuva-Fil ${ }^{\circledR}$, Experimental UV \# $1^{\circledR}$ and Experimental UV \#2 ${ }^{\circledR}$ ultraviolet-activated composite resins in monkey teeth to determine their biological compatibility with the dental pulp. The use of ZOE and silicate served as controls to establish baseline criteria for evaluation.

It is interesting to note that the pulpal 
GRAPH 2

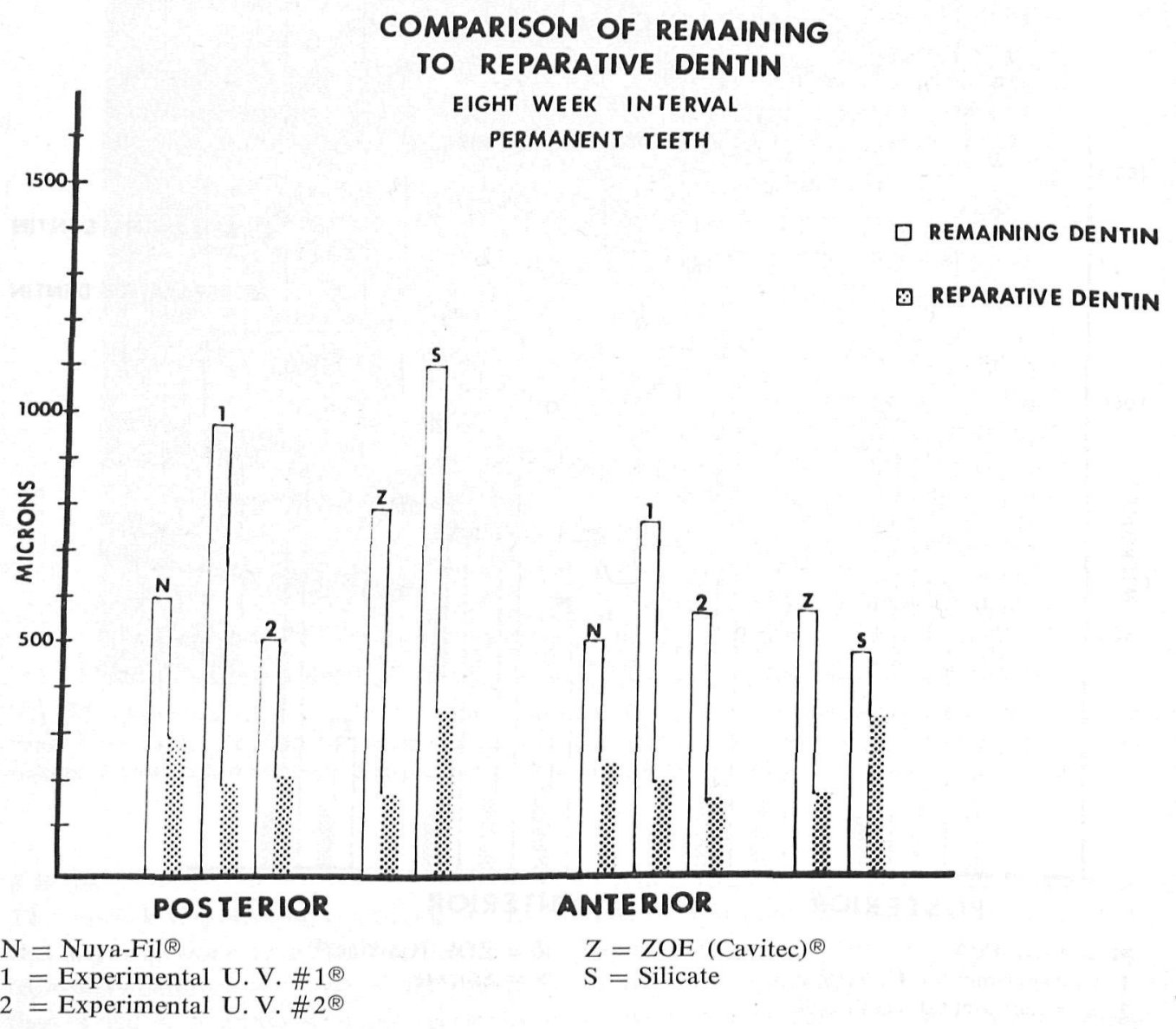

response for all three ultraviolet composites appeared comparable to $\mathrm{ZOE}$ and much less intense than that noted with silicate. Experimental data on pulpal response generally indicated that both ultraviolet and chemically-activated composite resins elicit more severe pulpal response than does ZOE. Stanley et al. (1972) found that after 60 days the ultraviolet-cured materials presented persistent or increasing responses as compared to a self-curing composite and ZOE, a fact we did not observe. They speculated that gradual increased pulpal

response was due to the incomplete cure of the material from one exposure to the ultraviolet light, resulting in irritating agents leeching out into the pulp. Our results indicated a slight pulpal response. It is possible that our slight response was due to the effectiveness of the cure of these materials resulting in a totally polymerized material preventing any leeching out of unreacted materials into the pulp, or conceivably that the materials used are not toxic to the pulp, regardless of whether a total or partial cure of these materials was achieved. Either of 
these factors may account for the slight response gained. Stanley et al. (1972) also noted the presence of focal hemorrhage in many of the specimens tested; $80 \%$ of ultraviolet and $33.3 \%$ of chemically-activated material showed focal hemorrhage at 3 days, while at 14 days the hemorrhage was no longer significant. Generally hemorrhage found in the pulp can be related to severe trauma to the tooth, such as a deep cavity preparation as reported in the studies by Seltzer \& Bender (1958). Stanley did not offer any explanation of this finding, only stating that further evaluation was needed. Our study did not show evidence of focal hemorrhage with any of these compounds at any time interval.

In reviewing the histograms (Figs. 12 and 13) it becomes obvious that, in spite of an attempt to standardize cavity preparation depth, there are still significant variations in the amount of remaining dentin. At both the 5- and 8-week time period, cavity preparations in anterior teeth tend to be deeper (that is less remaining dentin) than those noted in posterior teeth.

Reparative dentin measurements at 5 and 8 weeks indicate that there is a slight increase in reparative dentin formation as the post-operative time increases from 5 to 8 weeks.

At both 5 and 8 weeks the amount of reparative dentin for each time period is relatively similar, regardless of the amount of remaining dentin left, with the possible exception of silicate at 8 weeks. Generally there is a correlation between the amount of reparative dentin formed and the amount of remaining dentin left, that is, as the cavity preparation becomes deeper, a greater amount of reparative dentin is formed. This fact does not seem applicable in this study and it is difficult to explain why this is so. It may be that most of the pulpal irritation is due to cavity preparation and not the materials tested, or that even in our deepest cavity there was still sufficient remaining dentin to protect the pulp. There may be a more complete curing of the ultraviolet system with relatively inert filling material, resulting in slight pulpal irritation and reparative dentin formation. Whether there are feasible explanations or not is open to speculation. Further study in this area is needed to evaluate these parameters.

The histograms indicate that Nuva-Fil ${ }^{\circledR}$ produces a slightly greater amount of reparative dentin than do either Experimental UV \# $1^{\circledR}$ or Experimental UV \# $2^{\circledR}$. When we cured the Nuva-Fil ${ }^{\circledR}$ we used the Nuvalite and when we cured the Experimental UV \# $1^{\circledR}$ and Experimental UV \# $2^{\circledR}$ resins we utilized a Kerr photo light. There may have been a more complete cure with the new Kerr light, resulting in less available unreacted material leaking into the pulp, or the Nuva-Fil ${ }^{\circledR}$ may be slightly more toxic. Whatever the reason it is apparent that even though pulpal responses appear similar, there are differences in the amount of reparative dentin deposition.

In summary, our findings indicate that the pulpal response to the UV-activated composite resins is minimal and similar to ZOE. As was stated previously this is in contrast to previous research on both chemically cured and UV-activated resins. Continued development of less toxic materials or a more complete cure of these resins is undoubtedly the reason for such a minimal response. However, the work of Tobias et al. (1973)-on composite resins with and without a liner, indicates that composites used without a liner produced a substantially greater inflammatory reaction and reparative dentin deposition than the composite used with a liner. They therefore recommended the use of a protective liner in cavities restored with composites. In light of these findings we believe the placement of a liner is still recommended and advised under UV composites to minimize pulpal irritation. 


\section{Acknowledgements}

The authors wish to thank Miss Kathleen R. Engelhard, and Messrs. John and James Baker for their technical assistance.

\section{References}

Buonocore, M. G. (1970) Adhesive sealing of pits and fissures for caries prevention with the use of ultraviolet light. Journal of the American Dental Association 80, 324-328.

Council on Dental Materials and Devices: Recommended Standard Practices for Biological Evaluation of Dental Materials (1972) Journal of the American Dental Association 84, 382386.

Mjör, I. A. \& Tronstad, L. (1972) Experimentally induced pulpitis. Oral Surgery, Oral Medicine and Oral Pathology 34, 1, 102-108.

Seltzer, S. \& Bender, I. B. (1958) Early human pulp reactions to full crown preparations.
Journal of the American Dental Association 59, 915-930.

Stanley, H. R., Myers, C. L., Heyde, J. B. \& Chamberlain, J. (1972) Primate pulp response to an ultraviolet light cured restorative material. Journal of Oral Pathology 1, 108-114. Stanley, H. R., Swerdlow, H. \& Buonocore, M. G. (1967) Pulp reaction to anterior restorative materials. Journal of the American Dental Association 75, 132-141.

Tobias, M., Cataldo, E., Shierre, F. R. \& Clark, R. E. (1973) Pulp reaction to a resin bonded quartz composite material. Journal of Dental Research 52, 1281-1286.

Address:

R. J. Heys

Department of Operative Dentistry

Dental Research Institute

University of Michigan

Ann Arbor

Michigan 48109

U.S.A. 
This document is a scanned copy of a printed document. No warranty is given about the accuracy of the copy. Users should refer to the original published version of the material. 